\title{
Generation Analysis of Blockchain Technology: Bitcoin and Ethereum
}

\author{
Ms. Sidra Anwar \\ Memorial University of Newfoundland, St. Johns, Canada \\ Email: engrsid.es@gmail.com
}

\section{Sadia Anayat}

Govt. College women university, sialkot, Pakistan

Email: cssadiaanayat@gmail.com

\section{Sheeza Butt}

Govt. College women university, sialkot, Pakistan

Email: sheezabuttsheezabutt@gmail.com

\section{Saher Butt}

Govt. College women university, sialkot, Pakistan

Email: saherbuttsaherbutt814@gmail.com

\author{
Muhammad Saad \\ University of Gujrat, Sialkot Campus, Sialkot \\ Email: Saadchohan570@gmail.com
}

Received: 28 January 2020; Accepted: 25 March 2020; Published: 08 August 2020

\begin{abstract}
In this paper, the importance of blockchain technology have been discussed and the generations of blockchain (Bitcoin and Ethereum) have been compared provided different aspects. The blockchain is a technology which allows direct transaction without involving third party. Also, it offers many facilities like high translucency, high safety and security, improved trace-ability, greater proficient and transactions' speed, and reduced costs. Moreover, the cryptocurrencies provide advance security level. The basic purpose of this study is to highlight different aspects of Blockchain, Bitcoin and Ethereum and to show which cryptocurrency is better approach. The research contributes to show the impact of this technology in different fields and a comparison of bitcoin and ethereum is presented to analyze and furnish a decision regarding the best among them.

The use of blochchain technology in government applications can bring a drastic change in the world because it is safer and faster. Also, the comparison shows that ethereum is better than bitcoin as it is efficient and has more applications as compared to bitcoin. It offers more advanced services such as smart contracts. All in all, the analysis has concluded with Ethereum as faster and securer approach.
\end{abstract}

Index Terms: Cryptocurrencies, Blockchain, Ethereum, Bitcoin

\section{Introduction}

Recently, the phenomenon of crypto-assets has received much attention throughout the world. Crypto-assets are often called crypto currencies. The crypto currencies can be described as digital units and using cryptograph and these units are transfered between users. Whereas, Blockchain is a technology over which the crypto currencies operate. It was founded by Satoshi Nakamoto in 2009. This technology doesn't require any third trusted party for business operations [4]. Blockchain is widely used for engineering applications, avoiding the trust on central third party. It is a scattered directory that stores deals occurring in P2P network. It is decentralized, digitized and a open directory of all transactions of crypto currencies [2]. Blockchain has attracted much attention in recent years as basis of cryptocurrencies. Three technologies are used in blockchain, first is Cryptography of Private key then a network that is distributed and decentralized, including a shared ledger. And last one is method of accounting for all network related transactions and records. 
As Blockchain is unchangeable, so it is expected to bring the change in industry, commerce and drive global level change in economy enabling the certain and safe, quick and clear solutions that can be both private or public [6]. Blockchain technology has attracted the attention of many companies who want to add the distinct features of it to their security structures. It is a world wide network that allows individual or companies to cut out middle man while making transactions. During a transaction, by digitally signing the public key of next owner, each owner hand over a coin to next owner in blockchain and a hash of foregoing transaction. These are added to the end of the coin. The signatures are confirmed by the payee to confirm the ownership's chain. To deal with the issue of double spent, all transactions in this technology are announced openly and publicly and all participants are concurring on the archive of order in which they were experienced. In block, asymmetric Cryptography is used to secure the transaction between the users. In this network, each user has a public and private key. Public key give away no personal data so it can be shared with the other users in the network. As blockchain is decentralized and transaction' record is stored on multiple computers so it is not possible to hack this network. The working of blockchain is not so complicated. First of all, all newly recorded transactions are transmitted to all other node. Then the transactions that are new are collected into a block by all participating nodes. After collecting transactions, Nodes start work by finding the proof-of-work for its block. In fourth stage, node transmits block to all the other nodes. After that, all the other nodes support the block only if all transaction in the block are valid. At last, nodes begin to work on building the next block on chain to express their acceptance of block. They use hash of approved block as previous block for creating new blocks. A hash of block items is taking by timestamp server for time-stamping and then issue the hash such as in a Usenet post or newspaper. Thus a chain is formed as each previous timestamp is included in the hash of each timestamp. Proof of work scans the hashed values. Hash function then starts with bit number zero.

Blockchain is a network that uses cryptography to host applications and transfer digital instrument values from one person. Cryptography is an art of communication through coded message [8]. Bitcoin is first and still largest cryptoasset [1]. Bitcoin allows transferring money online between two parties without involving third party. This system protects the seller from fraud because transactions are computationally impractical to reverse. After introducing Bitcoin, new crypto-assets, Ethereum and Litecoin were also created according to same rules. Bitcoin and Ethereum work on separate blockchain [2]. Over the last couple of years, the number of crypto-assets has increased rapidly. Now there are almost 1500 crypto-assets.

This main objective of this study is to compare the bitcoin and ethereum to find out the best omong them. The comparison is made on the basis of different aspects which shows that ethereum technology offer more services than bitcoin.

This research is structured as follows: firstly, the background is discussed in section 3.1 and related work is presented in section 3.2. Furthermore, analysis is provided in section 4 and draws conclusion in section 5 . At the end the paper, the future work has been discussed.

\section{Methodology}

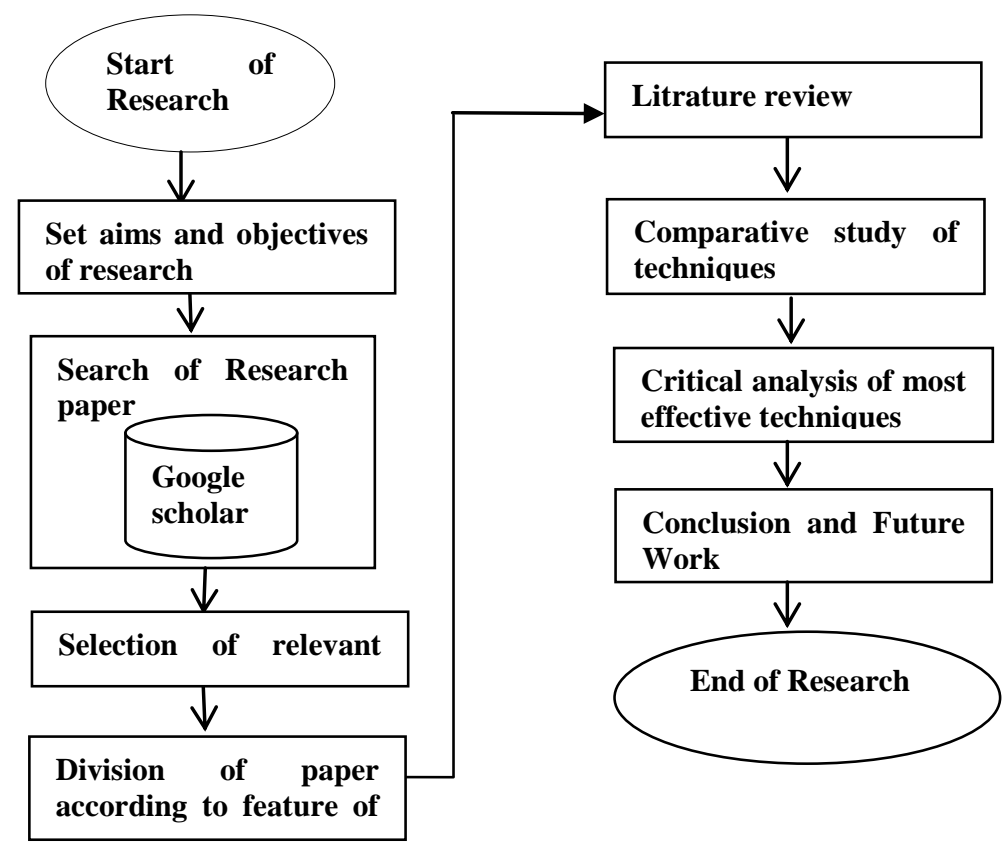

Fig.1. Methodology of conducting this study 
The basic goal of this study is to explore the knowledge of people about blockchain and the way this network works. This paper also covers some points of blockchain generations like bitcoin and ethereum. In first section, the literature review has been discussed. A small knowledge about blockchain and its generations (bitcoin, ethereum) is mentioned. Then comparison of blockchain, bitcoin and ethereum related to some aspect has been shown in form of table. Then result of comparison is discussed. At last, conclusion of whole study is defined.

\section{Litrature Review}

\subsection{Background}

Technical terms: First of all, the meaning of terms relating to blockchain technology must be clarified. The following table lists such terms and the meaning of those terms.

Table 1. Description of technical term used in blockchain

\begin{tabular}{|l|l|l|}
\hline Serial & Term & Description \\
\hline 1 & decentralization & $\begin{array}{l}\text { There is no need of central authority. Data is stored } \\
\text { across network on multiple computers. }\end{array}$ \\
\hline 2 & Immutable & $\begin{array}{l}\text { Once a transaction is performed in blockchain, it } \\
\text { cannot be changed or reversed. }\end{array}$ \\
\hline 3 & Transparent & $\begin{array}{l}\text { The ledgers that share decentralized network number } \\
\text { can be seen by everyone in the node. }\end{array}$ \\
\hline 5 & Minor & $\begin{array}{l}\text { For transaction, a date and time is used as an } \\
\text { eclectronic time stamp within a computer }\end{array}$ \\
\hline 6 & Hash function & $\begin{array}{l}\text { Minor is one who varify the transactions } \\
\text { hash function is used. }\end{array}$ \\
\hline 7 & Node & $\begin{array}{l}\text { In blockchain technology, nodes can be defined as } \\
\text { ledger }\end{array}$ \\
\hline
\end{tabular}

Blockchain: Before 2008, there were a lot of financial institutes which were serving as third party to perform the electronic payment. The commerce on internet had to rely on such financial institute. Although this system was working in a well-established way to perform transactions but still there were a lot of concerns unresolved. One of them was weakness of trust based model. This transaction institute couldn't avoid the dispute regarding mediation because non reversible transactions were not possible. The transaction cost increased because of mediation cost, cutting off possibility from casual transaction. There was no ability to make non-reversible payment from non-reversible services. The trust spread with possibility of reversible approach. A certain percentage of fraud was accepted as it cannot be avoided. By using tangible currencies, these uncertainties related to payment can be avoided in person. But there were no mechanism to transfer money directly without third party involvement.

What was required was a system that provides facility of electronic payment. Instead of trust, the system should be based on crypto-graphic tool. At the beginning of 2009, Blockchain was introduced and it was a technology on which all the crypto currencies operated further-on. The blockchain is digitized and not centralized; also the ledger of cryptocurrencies transactions is public in nature. In Blockchain, the record of data is distributed and managed by cluster of computers. It is peer to peer distributed timestamp server. In addition, blockchain technology is digitized and decentralized to generate the computation proof of sequential order of transactions. This system protects the seller from fraud because transactions are computationally impractical to reverse. The process of verifying transaction is called mining and the person who verifies the transaction is known as miner. There are three technologies which are used in blockchain.

Table 2. Technologies used in blockchian

\begin{tabular}{|l|l|l|}
\hline Serial & Technology & Description \\
\hline 1 & Cryptography & Cryptography of private key \\
\hline 2 & Distributed ledger & $\begin{array}{l}\text { Distributed and decentralized } \\
\text { network including a shared ledger }\end{array}$ \\
\hline 3 & Mean of accounting & $\begin{array}{l}\text { For the network related } \\
\text { transactions and documents }\end{array}$ \\
\hline
\end{tabular}


As this technology is redistributed so it cannot be hacked. It is safe and secure till a sincere nodes control more power of CPU collectively [3]. Blockchain is sets of data which are recorded permanently and provides a fast and cheaper way for cross border payment. Blockchain technology also provides the grate level of security. The security and verification processes can take place throughout computers which are member on blockchain network. In transaction cases, this verification is used to approve transactions before adding them into chain.

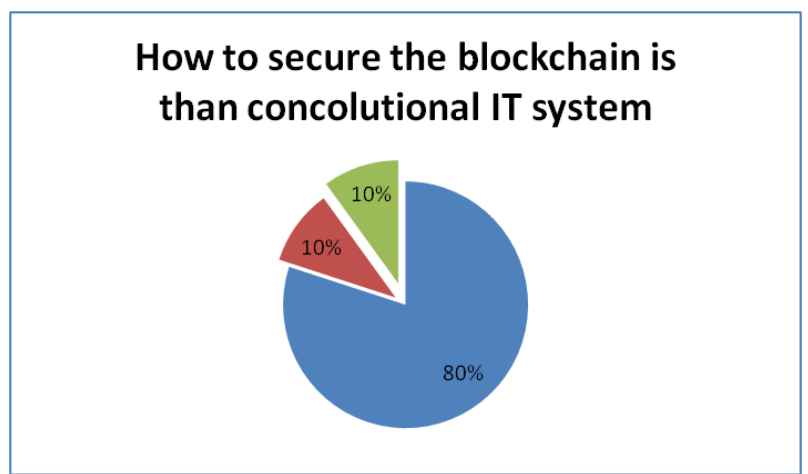

Fig.2. The security level of blockchain technology [1]

Table 3. Figure 2 analysis

\begin{tabular}{|c|c|c|}
\hline Grey: & Less secure & $10 \%$ \\
\hline Mustard: & No secure & $10 \%$ \\
\hline Blue: & secure & $80 \%$ \\
\hline
\end{tabular}

Bitcoin: it is the first and largest crypto-currency. Bitcoin generally deemed the first and the largest by market capitalization crypto currency. It was created in 2008 by a person or group behind the pseudonym of "Satoshi Nakamoto"[22] Bitcoin is a virtual type of money. Bitcoin is online version of cash and people can use it for buying or selling goods or services. But this trade can be made only from those shops who accept bitcoin. Provided this, Transaction can take place between two parties without involving any third trusted party. Bitcoin is a P2P system which is decentralized and do not require any monetary authority influence [5]. These transactions are verified by network nodes. Verified transactions are added to publicly distributed ledgers by Minors. These Minors are appreciated to do so by the reward the new bitcoin transaction fees. In last few years, Digital currencies like bitcoin have been adopted by a lot of people. Bitcoins are created using a computer. The people get Bitcoins by using the real money; also even get bitcoin by selling products and letting the people to pay them in bitcoin. Experts predict that there were 5 million users of bitcoin in 2019 [7]. Bitcoin is the first blockchain created by Satoshi Nakamoto in 2009. All the transactions of bitcoin are traceable and public. These transactions are stored permanently in the bitcoin network so it is not possible to make some change in those transactions. As bitcoin is decentralized and transaction' record is stored on multiple computers so it is not possible to hack this network. Bitcoin's addresses are the information that can define only where bitcoin is allocated where the bitcoin are sent. By each user's wallet, their addresses are created privately to hide information from the others. There are several possible ways which have been adopted by users to turn bitcoin into cash and move it to the bank account like Coinbase and Karken. If someone wants to sell his coin and withdraw resulting cash directly to the bank account then this is the best and easiest way. Bitcoin is the revolution of money so in future it will be much bigger then internet revolution because internet is only the communication revolution lately. Bitcoin will be arising as future money. Amazon has also been started to accept bitcoin in their transaction which shows that bitcoin will become a mode of future finance [7]. 


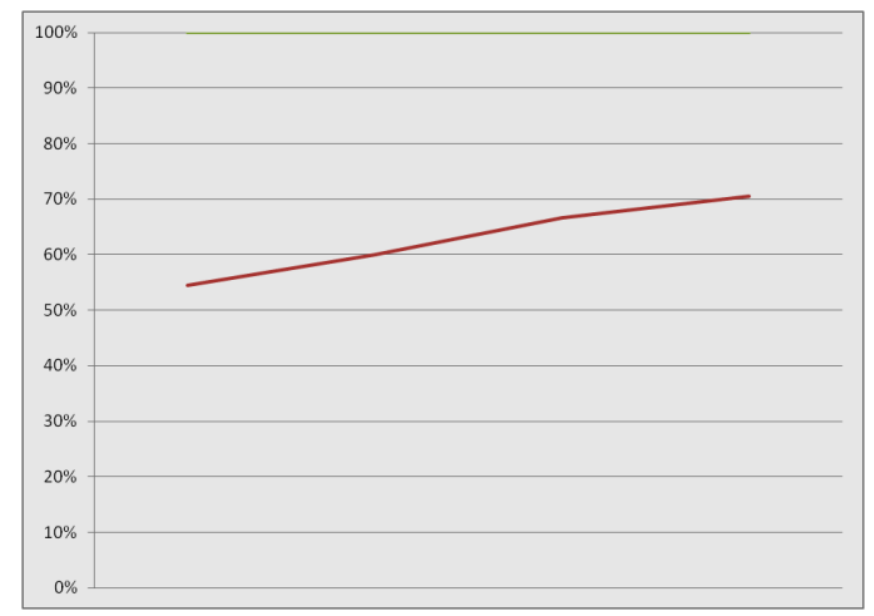

Fig.3. Bitcoin votality from january 2018 to december 2019 [7].

Basically each bitcoin is a computer file. "Digital wallet" app is used to store the bitcoins on mobiles or PCs. People can send and receive Bitcoin (or part of bitcoin) to digital wallets. Blockchain is used to keep record of every single transaction. Because of this record, it becomes possible to trace history of bitcoin. It helps to cessation of using the coins they do not own. Bitcoin can be generated in multiple ways. Bitcoins may be generated by powerful computers with high consumption of electricity. The people may make their computers to process the transactions for everybody to make the bitcoin network to work. These systems are made to perform incredibly difficult sum. The owners of these systems are occasionally appreciated by rewarding with a bitcoin. In mining, people make their systems powerful just to try and get the bitcoin. Those people are called minors. To stop generating too many bitcoins, the sums are becoming more and more difficult. If someone starts mining now it could be year before he got a single bitcoin and might possible he could end up spending money on electricity for his system than the bitcoin would be worth. Brain wallet is enabling enough to recover the private key.

Ethereum: In late 2013, Vitalik Buterin founded the ethereum. It is the second-generation of the blockchain technology. Ethereum is an underlying block chain and ether is currency of Ethereum. It is second largest crypto currency. Ether was introduces in 2015.

The features of Ether are unique different from the bitcoin. The smart contracts are the major innovation that brought about by Ethereum. In every n12 second, a new block is discover in ethereum network [23]. The transaction processing system of ethereum consist of a set of all accounts and their balances, a transaction and an updated set of accounts. Three different things can be referred by ethereum. Firstly, one is ethereum protocol which is derived from the bitcoin, secondly, it is ethereum network that is created by computers using ethereum protocols and lastly, it is Ethereum project funding development [8]. The main focus of Ethereum blockchain's principle emphasis is to run on the programming code of any decentralized application. In the Ethereum blockchain, ethers are earned by minors instead of bitcoin. Block headers are block of information in ethereum [9]. The Ethereum network offers pseudoanonymity. Its distributed nature and transparency enable many radical new technological capabilities. The state tree is used to store the balance of an account address and change whenever transactions in opposition to that particular account occur. It makes sense that permanent data should be stored separately. Ethereum provides guarantees about execution and integrity of data for smart contracts. Smart contract are more powerful application of blockchain technology that supervises all aspects of any agreement from start to end. When condition becomes true, the smart contracts can be self-enforcing and self-executing.

It is assumed that before execution, all transactions first have to pass initial test of intrinsic validity. It may include to check that transitions are well formed RLP, and there is no additional trailing byte. Then Signature and nonce of transaction is valid. Moreover, Intrinsic gas is no larger than gas limit go used by transaction. At last, there must be a least standard cost in sender's account balance.

\subsection{Related Work}

Crypto assets are usually defined as digital units that are transferred between users by using cryptography. This study [1] focuses on how the money is different from crypto assets. Bitcoin is largest and first crypto asset which was created at the beginning of 2009. After introducing bitcoin, other crypto assets, like ethereum and litecoin were created according to same rules. In this research paper, the concept of Metalism, Chartalism and Functionlism is discussed. All crypto currencies are based on blockchain technology which is decentralized. Blockchain actually is a chain of blocks. In order to continue the tasks that are necessary for the system to perform function, the user will have a motive to get the profit. There are two main problems in the network of bitcoin. Firstly, the miners that first received a specific amount of newly created Bitcoins. Secondly, a person transfer bitcoin to someone else; and the miner receives a reward too. This study proved that crypto assets cannot be classified as money [1]. 
Different factors play an important role in geographic distribution of cryptocurrency nodes all around the world. This research has shows that electricity price is not much important in geographical distribution of crypto currencies and relationship between bitcoin and tor rely has a greater impact [2].

In October 2008, a new concept of peer to peer network was presented by Satoshi Nakamoto. According to this concept, transaction between two persons can be placed without involving the third party. Proof of work is used to make a record of all transaction. This technology is decentralized. When a transaction takes place in blockchain technology, the nodes in network check validity of that transaction. If majority of nodes is agreed on validation of transaction, then block of data is added into chain and it is visible to all nodes in network. The data is stored in public ledgers [3].

A comparison between the execution services of blockchain and cloud system was performed. In the software system development, the use of blockchain is an architectural choice. Blockchain is decentralized and distributed ledger. Ethereum is a technology based on blockchain and uses Turing complete scripted language for smart contracts. Blockchain can be used for execution of processes in two ways. First one is Choreography monitor, blockchain technology record the status execution of all processes for all nodes on blockchain. Second is Trigger that creates a link of process that is executing on blockchain with external world [4].

Different methods are used in this study and conclusion is that bitcoin is in the bubble phase. Relationship of bitcoin and ethereum with blockchain position and association between prices of both cryptocurrencies proved that there are different short time periods in which fundamental, hashrate and liquidity, effect price dynamic Ethereum and Blockchain [5].

The encyrption process of the blockchain transactions was defined. Whenever a transaction is added into a ledger, it is encrypted. Using encryptographic algorithm the transactions are encrypted and checked by all other systems in the network. After verification, if majority of computers are agreed on validation of transaction, the data block is connected to the chain and exchanged by all other systems in the network [6].

In this paper, the initial discoveries about motivation of people for pursuing the blockchain technology are reported. The Human Computer Interaction community has a greater interest in materializing and understanding the main concept of bitcoin. In order to identify the future direction that human computer interaction community may involve with, the user experience and motivation of using Bitcoin technology is being explored in this paper. The future direction that is explored in this paper is materiality of bitcoins and viability of technological involvement supporting it [7].

This study provides the detailed knowledge of bitcoin and ethereum technology. Bitcoin works like a LAN network. The working of ethereum is same as bitcoin works but the difference is, it supports Turing Computer scripting language. In order to make a P2P network that is accessible using web browser, a special software library is required to connect the front end of application with the back end. Another approach for development of software is offered by Ethereum where safety, privacy and trust are provided at protocol level. The wallets are the software that holds the keys to the ethereum virtual machine. Ethereum virtual machine offer functionality like fed-wire and a cluster of other facilities [8].

The proposed solution shows that the point where the latency is limited by the network diameter, it is possible to increase the scalability of protocol of blockchain. Ethereum is a decentralized network based on blockchain technology. It offers a technology where concept of all transaction-based state machines may be built. It includes the concept of smart contract where the payment is done if certain conditions become true [9]. In this paper, a new blockchain protocol, named Bitcoin NG is presented. Different metrics of interest are considered in measuring the safety and efficiency of bitcoin-like blockchain protocol. Using unchanged clients of both protocols, Large-scale experiments are performed at $15 \%$ the size of the operational Bitcoin system [10].

A brief overview of blockchain, bitcoin and ethereum is provided in this paper. Blockchain is a peer-to-peer network where a true mechanism is promoted based on concurrence of majority of computers. In the last few years, multiple cryptocurrencies and hashing techniques are rapidly growing. The Bitcoin ledger can be defined as a state transition system. The result of the state transition system is in the form of a new state. All transactions in bitcoin are determined with set of input and output and their hash values. Ethereum addressed multiple limitation of bitcoin technology. It supports computation of all types. Ethereum blocks contain block number as well as most recent state and the transaction list [11].

The working of bitcoin is explained in this study. Bitcoin is a virtual currency and can be issued by a company or even by an individual but issuer does not work under the government supervision. Bitcoin is decentralized cryptocurrency and virtual currency scheme. The flow of this virtual currency scheme is bidirectional. Software is used to make the bitcoin payment. That software is called "Wallet". Bitcoin is not a type of digital cash. When a transaction takes place, the payment does not occur in the form of note or coin, but the recipient's account is credited and sender's account is debited. But bitcoin is not suitable for all type of payments. It takes much time for the user to be sure that transaction has been added into blockchain [12].

Multiple etheruem security patterns have elaborated in this paper. An extensive pattern language is created where Ethereum is a cryptocurrency based on blockchain technology that provides the functionality of smart contract. The computation and status of all contacts in handle by Ethereum Virtual Machine. Smart contracts are employed on blockchain ledgers. These applications are executed as a part of validation. When the contract has been deployed, then problem of lack of transaction control can be solved by using the pattern that is presented in this study. The programs 
on Ethereum can be executed autonomously. But there are some issues as well. These problems may appear in the form of harmful callbacks or issue may arise as uncontrollable financial risk at stake. In future, the already collected pattern can be extended to create an informative design pattern language for Solidity [13].

A first standard of current applications and quick execution of different applications with larger RAM is provided[14].

A model of consumer acceptance and use of crypto currencies is presented here. Since early 2013, when market capitalization and volume of transaction became material, the bitcoin cryptocurrency has attracted great attention in public press. The fundamentals of market that determine exchange rate of in conjuctive currency to Bitcoin can be analyzed by using theoretical models. By 2015, the greater bitcoin balance is held by Asia than Europe and Easter Latin America. In 2013, the bitcoin was most famous and popular exchange in china. The ledger of bitcoin is decentralize and public. The translation of ledger data into economical quantities become challenging because of Different features of the system [15].

AsicBoost was intoduced that is based on a different and new way to process the task items in and outside of bitcoin mining. The bitcoin mining speed can be increase by the factor of approximately $20 \%$ by using the AsicBoost. This method is applicable to all types of hardware use for bitcoin mining. The hash function that is used for bitcoin mining is SHA 256 that is defined by NIST. In this hash function, massages are divided into chunks each chunk is of 64 bytes. A state machine is used to execute each chunk [16].

Vitalik Buterin presented a new cryptocurrency called ethereum. Bitcoin was first cryptocurrency introduced by Satoshi Nakamoto. It is still largest cryptocurrency but now attention is moving toward a new cryptocurrency known as Ethereum. Ethereum work just like bitcoin but difference is it offer "Smart contract" because it has built in turning language. There is a need of transaction fees because every published transaction forces the cost of needing to verify and download transaction on network. Any type of computation, including loop, can be encoded through Ethereum virtual machine code. A simple version of GHOST, consisting of five levels, is implemented by Ethereum[17]

The sales of Blockchain are based on digital token which are related with particular platforms. The initial coin offering are block chain sales. In this study, relationship between issuer and success measures with complete hub on assets by using initial coin offering was considered. The solution that we found is liquidity is higher when the institute issue tokens and they also assure that they create workable business platform. The tokens are also successful when they have fundamental useful functions [18].

A digital based currency takes hold of cryptograpy for its safe authenticity which acting as a moving continuously medium for financial transaction. Digital assets industry is straightly going through a phase of quandary. These quandary have created many problems for the once flourish digital assets industry in every country. The main threat the countries was facing is that people convert their savings into crypto currency. Many countries are trying to reduce the crimes like money laundering, terrorism. For this reason many countries banned cryptocurrency because they notice the threat to economy. Further studies can include information that how other companies have been working to make bit coins a legitimate mode of exchange and payments [19].

This paper was conducted to understand the factors which convert bit coins that are obtaining momentum in many fields of international finance and how unruly it can be, replacing main injunction currencies in financial system influence USD. It will not transform the process by which payments are made. It is possible to impact the world future currencies. USD is facing many dare from EURO. The need of crypto adds a new measurement to this challenge for USD. The conclusion is that the regulator does not motivate the usage of bit coin but we cannot ignore the technology because the crypto currencies are replacing with fiat currencies [20].

A system was implemented for e-voring by using ethereum blockchain. E-voting is a secure system for voting because it not allowed the duplication of votes and it is completely transparent system. it also protects the privacy of participant. The blockchain of ethereum holds all the records and votes. By using this system, we successfully move Evoting in blockchain platform. In politics and scientific circles E-voting is still considered as a disputed topic [21].

\section{Analysis}

Bitcoin and ethereum are the cryptocurrencies based on the blockchain technology. But both of these are different from each other. Bitcoin is just a currency whereas the ethereum is a ledger technology used to develope the new programs by companies. Here is the difference between bitcoin and ethereum

Table 4. Comparison between bitcoin and ethereum

\begin{tabular}{|l|l|l|l|}
\hline Serial & Difference & Bitcoin & Ethereum \\
\hline 1 & privacy & $\begin{array}{l}\text { Decentralized store of value, a peer-t- } \\
\text { peer digital currency }\end{array}$ & $\begin{array}{l}\text { Decentralized smart application that } \\
\text { cannot altered, ZK notes }\end{array}$ \\
\hline 2 & 7 & 15 \\
\hline 3 & Transaction & Blockchain & Blockchain \\
\hline 4 & Technology & Hardware wallet, Cold storage wallet & Stack, Memory, non-volatile storage \\
\hline 5 & Storage & 10 minute & 15 second \\
\hline 6 & Average block time & 1 mega byte & 10 to $20 \mathrm{~kb}$ \\
\hline
\end{tabular}




\begin{tabular}{|c|c|c|c|}
\hline 7 & Votality & $\begin{array}{l}\text { Bitcoin are more volatile than traditional } \\
\text { currencies }\end{array}$ & $\begin{array}{l}\text { Etheruem closes in on the lowest volatility } \\
\text { of all time }\end{array}$ \\
\hline 8 & Smaller transaction & Satoshi & Wei \\
\hline 9 & Peak price & $\begin{array}{l}\text { In early janaury 2018, Bitcoin price } \\
\text { peaked at about } \$ 20000\end{array}$ & $\begin{array}{l}\text { In early janaury 2017, ethereum price } \\
\text { peaked at about } \$ 1400\end{array}$ \\
\hline 10 & Minniig & $\begin{array}{l}\text { Bitcoin Minning is base on proof of } \\
\text { work }(\mathrm{PoW}) \text { alogorithm }\end{array}$ & $\begin{array}{l}\text { Ethereum Minning is base on proof of } \\
\text { stake(PoS) alogorithm }\end{array}$ \\
\hline 11 & Application & $\begin{array}{l}\text { Bitcoin is pitched as digital currency, } \\
\text { Medium of exchang }\end{array}$ & $\begin{array}{l}\text { Platform that facilitate immutable, } \\
\text { programmatic peer to peer contract and } \\
\text { application via its own currency }\end{array}$ \\
\hline 12 & Future importance & $\begin{array}{l}\text { By the june } 2020 \text {, Implied volatility of } \\
\text { bitcoin's is set to rise to over } 70 \% \text {, up } \\
\text { from } 55 \% \text { for the middle of December } \\
2019\end{array}$ & $\begin{array}{l}\text { By the end of } 2020 \text {, the ethereum is } \\
\text { expected to more than double, to } \$ 1488\end{array}$ \\
\hline 13 & Popular alternate & Bitcoin cash $(\mathrm{BCH})$ & Ethereum classic(ETC) \\
\hline 14 & $\begin{array}{l}\text { Ammount of unmined } \\
\text { cryptocurrencies }\end{array}$ & $\begin{array}{l}\begin{array}{l}\text { There are } 17 \\
\text { circulation }\end{array} \\
\text { cillion bitcoin in }\end{array}$ & $\begin{array}{l}\text { there are more than } 100 \text { million Ether in } \\
\text { circulation }\end{array}$ \\
\hline 15 & Access mode & Public & Public \\
\hline 16 & Supply limit & There are total 21 millions bitcoin & There is no limit on its total coin supply \\
\hline
\end{tabular}

The above comarison of bitcoin and ethereum technology shows that both technologies are decentralized are based on blockchain. Bitcoin are stored in hardware wallets and stack or nonvolatile memory is used to store the ethereum. But ethereum somehow is better than bitcoin as there is no limit on its total coin but only 21 million bitcoins are there in the world. The ethereum perform more services than bitcoin does. Smart contract is a special feature of this cryptocurrency. Smart contacts can be used for many more things than one can use in Bitcoin. It is clear from above comaprison that ethereum transaction speed is fast than bitcointransaction speed as it take just 15 second to perform a transaction but bitcoin require 10 minutes to perform a transaction. Transaction fees of ethereum are cheaper than Bitcoin's transaction fees. If bitcoin was version 1.0, then Ethereum was version 2.0 that makes it possible to create on the top of it decentralized applications. The trading and market of Ethereum is occurring very rapidly with since it is only setting its price and demand trend.

\subsection{Usage of blockchain over existing systems}

A lot of applications of this distributed technology have developed in different countries to improve publicly provided government services [24].

Table 5. Usage of blockchain over existing systems

\begin{tabular}{|l|l|l|}
\hline Serial & System & Percentage \\
\hline 1 & $\begin{array}{l}\text { Faster speed as compare to } \\
\text { existing system }\end{array}$ & $32 \%$ \\
\hline 2 & $\begin{array}{l}\text { New business models and } \\
\text { revenue sources }\end{array}$ & $28 \%$ \\
\hline 3 & High security/lower risk & $21 \%$ \\
\hline 4 & Lower costs & $16 \%$ \\
\hline 5 & $\begin{array}{l}\text { No non perceived benefits } \\
\text { realtive to existing systems }\end{array}$ & $2 \%$ \\
\hline 6 & Other/not sure & $1 \%$ \\
\hline
\end{tabular}

\section{Discussion}

Blockchain Technology is one of the most consistent technologies when it requires keeping track of financial properties. The applications of Blockchain technology are not limited only to the finance industry [3]. It has a fantastic future in different sectors such as supply chain management, digital advertising, forecasting, cyber security, Internet of things, networking, etc [9, 11]. Bitcoin is first and largest cryptocurrency that has attracted much attention since last decade. It is based on blockchain technology. Moreover, in bitcoin, the transaction speed is low. While Ethereum is a cryptocurrency approach that increases transaction speed [17]. In addition, smart contract is a special feature of this cryptocurrency which can be used for many more things than one can use in Bitcoin. The Ethereum's blockchain was introduced in 2015 while Bitcoin was introduced in the beginning of 2009 [1, 3]. Therefore, it can be said that the blockchain of ethereum is another advanced version of blockchain of bitcoin. It solves some of its problems and a new feature, such as smart contracts, is introduced in this blockchain. Transaction fees of ethereum are cheaper than Bitcoin's transaction fees. The ethereum transaction speed is faster than bitcoin transaction speed. Bitcoin is nothing more than money whereas ethereum is a database that is used by businesses to build new programs. If bitcoin was version 1.0, then Ethereum was version 2.0 that makes it possible to create on the top of it decentralized applications. 
This study contributed to show the significance of blockchain technology in multiple systems. Bitcoin and Ethereum technologies are discussed in detail. Both technolgies are decentralized and based on blockchain technology. In this research, a comparison is performed on the basis of different aspect which concluded that ethereum technology is faster and cheaper than bitcoin. Moreover, it offer advanced services such as smart contracts. As the blockchain technology is fast and secure so the use of this technology in government applications can change the world drastically.

\section{Conclusion}

Blockchain Technology is one of the most consistent technologies when it requires keeping track of financial properties. The research work has shown that Ethereum's blockchain is advanced version of Bitcoin'blockchain. This study has given the better picture of which cryptocurrency is better and how the blockchain technology affects the existing businesses. In this paper, the ethereum and bitcoin are compared with respect to their common characteristics which was concluded with that ethereum is faster than bitcoin. As compared to bitcoin which was launched in 2009 , the ethereum is 5 years younger than bitcoin. This research contributed to highlight the difference between bitcoin and ethereum to show which one is better and why. In this paper, it is proved that the usage of blockchain technology in government sector and applications can bring a drastic change in the world.

\section{Future Work}

The above research have shown that Blockchain technology has a fantastic future in different sectors such as supply chain management, digital advertising, forecasting, cyber security, Internet of things, networking, etc. In future, Implementing Blockchain technology in government system can make their operations much more secure and efficient.

\section{References}

[1] Söderberg, Gabriel. "Are Bitcoin and other crypto-assets money?." Economic Commentaries 5 (2018): 14.

[2] Brown, Keaton. "Bitcoin and Ethereum: Empirical Evidence on Node Distribution." (2018).

[3] Nakamoto, Satoshi. Bitcoin: A peer-to-peer electronic cash system. Manubot, 2019.

[4] Rimba, Paul, et al. "Comparing blockchain and cloud services for business process execution." 2017 IEEE International Conference on Software Architecture (ICSA). IEEE, 2017.

[5] Corbet, Shaen, Brian Lucey, and Larisa Yarovaya. "Datestamping the Bitcoin and Ethereum bubbles." Finance Research Letters 26 (2018): 81-88.

[6] Crosby, Michael, et al. "Blockchain technology: Beyond bitcoin." Applied Innovation 2.6-10 (2016): 71

[7] Khairuddin, Irni Eliana, et al. "Exploring motivations for bitcoin technology usage." Proceedings of the 2016 CHI Conference Extended Abstracts on Human Factors in Computing Systems. 2016.

[8] Dannen, Chris. Introducing Ethereum and Solidity. Vol. 1. Berkeley: Apress, 2017

[9] Wood, Gavin. "Ethereum: A secure decentralised generalised transaction ledger." Ethereum project yellow paper 151.2014 (2014): 1-32.

[10] Eyal, Ittay, et al. "Bitcoin-ng: A scalable blockchain protocol." 13th \{USENIX\} symposium on networked systems design and implementation (\{NSDI\} 16). 2016.

[11] Vujičić, Dejan, Dijana Jagodić, and Siniša Ranđić. "Blockchain technology, bitcoin, and Ethereum: A brief overview." 2018 17th international symposium infoteh-jahorina (infoteh). IEEE, 2018.

[12] Segendorf, Björn. "What is bitcoin?." Sveriges riksbank economic review (2014): 2.

[13] Wohrer, Maximilian, and Uwe Zdun. "Smart contracts: security patterns in the ethereum ecosystem and solidity." 2018 International Workshop on Blockchain Oriented Software Engineering (IWBOSE). IEEE, 2018.

[14] Courtois, Nicolas, Guangyan Song, and Ryan Castellucci. "Speed optimizations in Bitcoin key recovery attacks." Tatra Mountains Mathematical Publications 67.1 (2016): 55-68.

[15] Athey, Susan, et al. "Bitcoin pricing, adoption, and usage: Theory and evidence." (2016).

[16] Hanke, Timo. "Asicboost-a speedup for bitcoin mining." arXiv preprint arXiv:1604.00575 (2016).

[17] Buterin, Vitalik. "A next-generation smart contract and decentralized application platform." white paper 3.37 (2014).

[18] Howell, Sabrina T., Marina Niessner, and David Yermack. Initial coin offerings: Financing growth with cryptocurrency token sales. No. w24774. National Bureau of Economic Research, 2018.

[19] Kheskani, Ashish, et al. "A Study On Cryptocurrencies and Its Issues."

[20] Seetharaman, A., et al. "Impact of Bitcoin as a world currency." Accounting and Finance Research 6.2 (2017): 230-246.

[21] Yavuz, Emre, et al. "Towards secure e-voting using ethereum blockchain." 2018 6th International Symposium on Digital Forensic and Security (ISDFS). IEEE, 2018.

[22] Kotane, Inta, et al. "Latgale National Economy Research."

[23] Triantafyllidis, Nikolaos Petros, and T. N. O. Oskar van Deventer. Developing an Ethereum blockchain application. Diss. Ph. D. Thesis, University of Amsterdam, Amsterdam, The Netherlands, 2016.

[24] Sari, Arif, and Seyfullah Kilic. "Exploiting cryptocurrency miners with oisnt techniques." Transactions on Networks and Communications 5.6 (2017): 62-62.

[25] Georgen, Christopher James, et al. "Method and Apparatus for Processing Mobile Payment Using Blockchain Techniques." U.S. Patent Application No. 15/839,280. 


\section{Authors' Profiles}

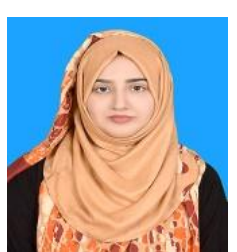

domains in IOT.

Sidra Anwar was born in Sialkot, Pakistan in 1992. She is a $\mathrm{PhD}$ scholar in Memorial University of Newfoundland, Canada. Previously, she has been a lecturer in Computer Science at the Govt. College Women University, Sialkot, Pakistan and member of the ORIC Steering committee since 2015. She did her BS in Software Engineering from Fatima Jinnah Women University, the Mall, Rawalpindi, Pakistan and MS in Project Management from COMSATS Institute of Information Technology, Islamabad, Pakistan. She is also a Registered Engineer under Pakistan Engineering Council Act 1976 with Registration\# COMP/14954. She has personally worked and supervised many projects regarding decision support systems serving multidisciplinary

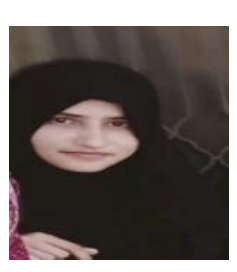

Sadia Anayat was born in Sialkot in 1998. She did her matriculation level in 2014 from, Govt. Girls Higher Secondary school Sambrial, district Sialkot in Science Subjects and her intermediate level (I.C.S) in 2016 from Superior college Sambrial, district Sialkot. Now she is doing her BS (Hons) in Computer Science (CS) from GCWU Sialkot. She is also certified as Microsoft Office specialist. Currently she has been working on research in Blochain technology and comparison between bitcoin and ethereum topic. Her main areas of research interest are blockchian technology.

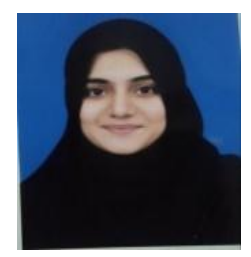

Sheeza Butt was born in Sialkot in 1999. She did her matriculation level in 2014 from, Govt. Girls Higher Secondary school Sambrial, district Sialkot in Science Subjects and her intermediate level (I.C.S) in 2016 from Superior college Sambrial, district Sialkot. Now she is doing her BS (Hons) in Computer Science (CS) from GCWU, Sialkot. She is also certified as Microsoft Office specialist. Currently she has been working on research in Blochain technology and comparison between bitcoin and ethereum topic. Her main areas of research interest are blockchian technology.

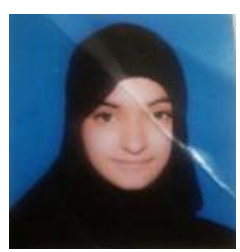

Saher Butt was born in Sialkot in 1999. She did her matriculation level in 2014 from Danish Public High School Sambrial, Sialkot in Science Subjects and her intermediate level (I.C.S) in 2016 from Superior College Sambrial, Sialkot. Now she is doing her BS (Hons) in Computer Science (CS) from GCWU, Sialkot. She is also certified as Microsoft Office specialist. Currently she has been working on research in Blochain technology and comparison between bitcoin and ethereum topic. Her main areas of research interest are blockchian technology.

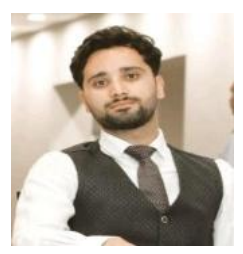

Muhammad Saad received his degree of BS in Software Engineering in 2019 from University of Gujrat. Recently, he is conducting research in Cloud Computing, Blockchain technologies and Artificial intelligence.

How to cite this paper: Sidra Anwar, Sadia Anayat, Sheeza Butt, Saher Butt, Muhammad Saad, " Generation Analysis of Blockchain Technology: Bitcoin and Ethereum", International Journal of Information Engineering and Electronic Business(IJIEEB), Vol.12, No.4, pp. 30-39, 2020. DOI: 10.5815/ijieeb.2020.04.04 